\title{
Long-term repetition-frequency stabilization of all-normal- dispersion Yb-doped fiber laser to the cesium standard
}

\author{
C. Ülgüdür ${ }^{1}$, F. Ö. Ilday ${ }^{1}$ and R. Hamid² \\ ${ }^{1}$ Department of Physics, Bilkent University, TR-06800, Ankara, Turkey \\ ${ }^{2}$ TÜBİTAK, Ulusal Metroloji Enstitüsü (UME), PO 54, TR-41470, Gebze-Kocaeli, Turkey
}

\begin{abstract}
Repetition-frequency stabilization of a Yb-doped fiber laser to the Cesium standard is reported. Laser amplitude and phase noise is characterized. Performance is limited to $2 \times 10^{-14}$ at 100000 averaging time by intrinsic stability of the Cs-standard.

(C)2010 Optical Society of America

OCIS codes: (320.0320) Ultrafast optics; (060.3510) Lasers, fiber; (120.3940) Metrology; (140.3425) Laser stabilization; (140.3538) Lasers pulsed; (320.7090) Ultrafast Lasers
\end{abstract}

Femtosecond laser frequency comb has revolutionized frequency metrology in recent years. While most of the initial activity utilized Ti:sapphire lasers, frequency combs generated from fiber lasers are increasingly important due to their advantages in long-term stability, cost-efficiency and operation-convenience [1]. Extremely long durations of uninterrupted operation are being reported from mode-locked fiber lasers. However, most of the activity is centered on Er-doped fiber lasers, with very few reports [2] utilizing Yb-doped fiber lasers, even though this gain medium offers superior technical performance in nearly every aspect. In particular, all-normal-dispersion (ANDi) lasers have been attracting much attention with their simplified cavity, increased ease-of-mode-locking and good technical performance [3]. However, to date, there is no study exploring the long-term stability of an ANDi laser, nor its use for comb generation. Here, we demonstrate for the first time to our knowledge, long-term (over several days) stabilization of an ANDi Yb-doped fiber laser to a Cs frequency standard. Characterization of short-term amplitude and phase noise characterization of the oscillator coupled with its long-term stability demonstrates its potential for use as a frequency-comb generator.

The $\mathrm{Yb}$-doped fiber laser has been constructed with a focus on its long-term stability and ease of transportation (Fig. 1(a)). The laser includes both a fast, piezo-based fiber stretcher and a slow, motorized translation stage to control the cavity length. The motorized stage is used to roughly set the repetition rate to the desired value and the fast stretcher is used for electronic locking to the reference. The repetition rate is $108.33 \mathrm{MHz}\left(12^{\text {nd }}\right.$ sub-harmonic of $1.3 \mathrm{GHz}$ ), and the laser produces $0.8 \mathrm{~nJ}$-energy chirped pulses, which can be dechirped to $127 \mathrm{fs}$. The optical spectrum is characteristic of this laser type (inset of Fig. 1(b)). The short and long-term stability of the laser is characterized in detail. The relative intensity noise (RIN) [4] of the laser is measured to be $0.072 \%$ within a bandwidth from $1 \mathrm{~Hz}$ to $250 \mathrm{kHz}$ (Fig. 1 (b)). As an indicator of the short-term frequency stability of the laser, its single-side band phase noise is characterized with direct photodetection (using a $12 \mathrm{GHz}$ photodetector, ET-3500 from Electro-Optics Technology) at $1.3 \mathrm{GHz}$ (corresponding to the $12^{\text {th }}$ harmonic of the repetition rate) using a Rohde \& Schwarz FSUP26 signal source analyzer (SSA). The measurement is limited by excess noise from the photodetection and the SSA and should be understood as an upper limit to the actual phase noise (Fig. 2(a)). The corresponding RMS timing jitter is $41 \mathrm{fs}$ from $1 \mathrm{kHz}$ to $30 \mathrm{MHz}$. The RF spectrum of the laser shows 100-dB suppression of the sidebands, which we believe to be caused by the laser power supply (Fig. 2(a)).
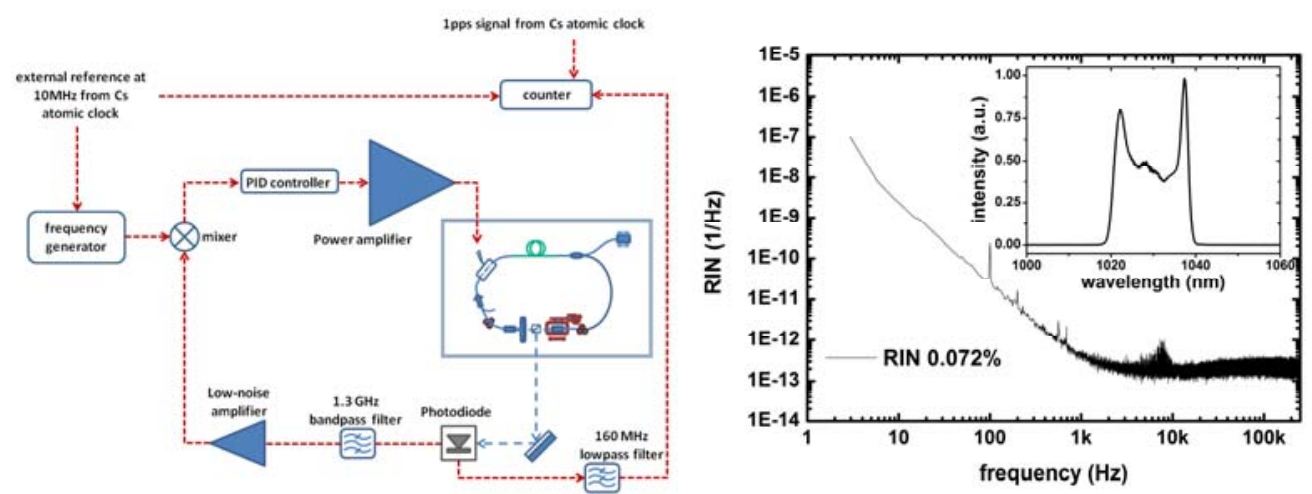

Fig. 1. (a) Overview of the experimental setup including schematic of the ANDi fiber laser and the PLL system. (b) Measured relative intensity noise (RIN) of the Yb-doped fiber laser, inset: optical spectrum of the fiber laser.

For long-term stabilization of the laser to the Cs reference, a phase-locked loop (PLL) system based on a PID controller is used (Fig. 1(a)). The error signal for the PLL, which acts on the piezo stretcher upon amplification, is generated by mixing the reference signal with the corresponding harmonic of the photodiode output of the laser (we typically use the harmonic at $1.3 \mathrm{GHz}$ ). The Cs atomic clock with high-performance tube (HP5071A) used in 
this study, located at UME, is a reference source for the UTC(UME) time scaling generation. Long-term frequency stability of the atomic clock is around $2 \times 10^{-14}$. The $10-\mathrm{MHz}$ reference signal from the Cs atomic clock at UME is up-converted to $1.3 \mathrm{GHz}$ through an externally Cs-standard referenced frequency generator. Upon locking the laser to the Cs-generated reference signal, the long-term stability performance fiber laser is characterized using Allan deviation [5]. The $1.3 \mathrm{GHz}$ electrical signal coming from the laser through an out-of-loop photodiodebandpass filter combination is measured with a time interval and frequency counter (SR620), which is externally triggered from the Cs standard. Instead of direct frequency count, time interval measurement method is preferred for improved fidelity. To this end, 1 pps signal from the same Cs-atomic clock is supplied to the counter to implement the phase difference count method.

During the experiments, the Cs clocks and the laser were in different rooms, and the $1 \mathrm{pps}$ and $10 \mathrm{MHz}$ outputs of the Cs clocks were transferred by $20 \mathrm{~m}$-long BNC cables. The long cable introduces additional phase noise. In Fig. 2.b, stability of the $10 \mathrm{MHz}$ output after the long BNC cable is given. As to be expected, usage of a long cable causes an increase in the white-noise level. While stability measurement next to two identical clocks with a $1 \mathrm{~m}-$ long cable yields a stability of $1.5 \times 10^{-11}$ after 1 second averaging, it increases to $6.8 \times 10^{-11}$ at $1 \mathrm{~s}$ averaging when using the 20 meter long cable. Nevertheless, with long-term averaging, this difference vanishes and a stability of $3.2 \times 10^{-14}$ is reached for both cases at 65000 seconds averaging times. In short, using 20 meter long cale in the actual experiment determines the background stability level of the results.

In order to determine effects of the atomic clock and the frequency counter on the stability measurements of the laser in the final setup, the frequency stability of two similar Cs atomic clocks were measured using a short cable in the same room. During this measurement, frequency counter was operated in time interval counter mode and externally triggered with the $10 \mathrm{MHz}$ reference output signal of the second similar atomic clock. 1 pps outputs of both the reference Cs atomic clock used in the laser stabilization experiment and of the other Cs atomic clock were fed to the counter to investigate their time difference through long-term Allan variance statistics. The results are given in Fig. 2(b). As expected, white noise is dominant and long-term stability is around $2 \times 10^{-14}$. This measurement method also reflects the stability of $10 \mathrm{MHz}$ RF signal output of the clock.
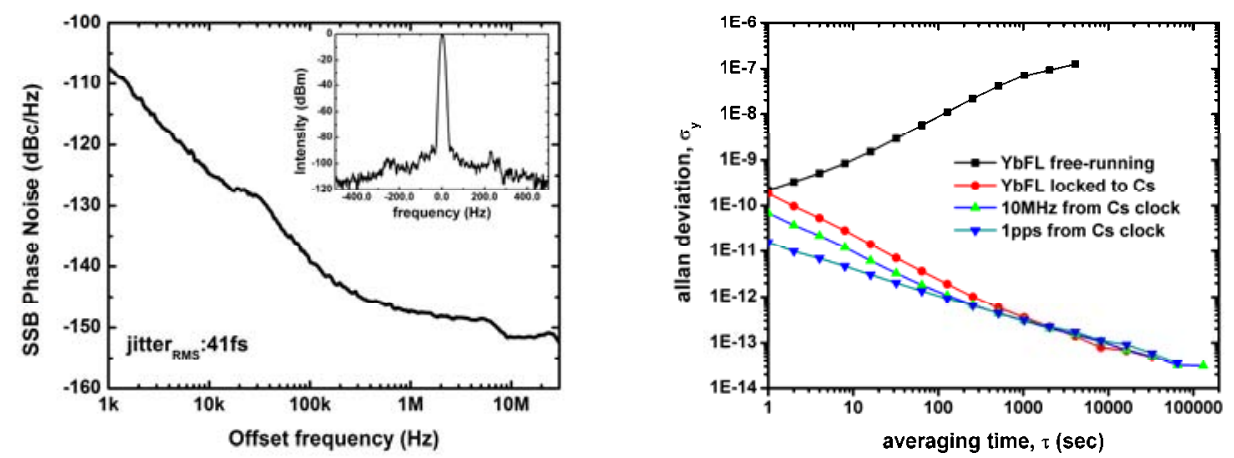

Fig. 2. (a) Single side band phase noise of the laser, inset: RF spectrum of the repetition frequency of the laser. (b) Allan deviation measurements of free-running YbFL, $10 \mathrm{MHz}$ RF sgnal from Cs atomic clock, 1 pps signal from Cs atomic clock and Cs-locked YbFL.

The long-term stability of the laser in both free-running and Cs-locked mode is given in Fig. 2 (b). For the freerunning operation, repetition rate stability is dominated by drift noise. Stability at 1 second averaging time is about $2 \times 10^{-10}$, increasing up to $1 \times 10^{-7}$ at 4000 seconds averaging times due to thermal drifts. When the repetition rate of the laser is locked to the $\mathrm{Cs}$ atomic clock, its stability increases with increasing averaging times, converging to the the Cs clock's stability after 1000 seconds averaging time. Until then, the stability of the laser is somewhat worse than that of the Cs atomic clock, which might be stemming from the PLL electronics. However, beyond 1000 seconds averaging times, the laser completely inherits the stability of the Cs clock, confirming that flicker and drift noise does not affect its stability in the long term.

In conclusion, we have demonstrated long-term stabilization of an all-normal-dispersion Yb-doped fiber laser to a Cs atomic clock for the first time. Allan deviation stabilities of $10^{-14}$ are obtained after one-day-long averaging, which suggests Cs standard-like performance of the laser. For these experiments, the laser was transported between two cities, after which it was mode-locked very easily and provided uninterrupted mode-locked operation and locking to reference during several days of experimentation. Given their simplicity and robustness, we expect the all-normal-dispersion $\mathrm{Yb}$-doped fiber laser to become a viable choice for frequency metrology applications.

[1] N. R. Newbury, and W. C. Swann, "Low-noise fiber-laser frequency combs (Invited)," JOSA B 24, 1756 (2007).

[2] T. R. Schibli, I. Hartl, D. C. Yost, M. J. Martin, A. Marcinkevicius, M. E. Fermann, and J. Ye, "Optical frequency comb with submillihertz linewidth and more than $10 \mathrm{~W}$ average power," Nat. Photon. 2, 55 (2008) and references therein.

[3] A. Chong, J. Buckley, W. Renninger, and F. W. Wise, "All-normal-dispersion femtosecond fiber laser," Opt. Exp. 14, 10095 (2006).

[4] I. L. Budunoğlu, C. Ülgüdür, B. Oktem, and F. Ö. Ilday, "Intensity noise of mode-locked fiber lasers," Opt. Lett. 34, 2516-2518 (2009).

[5] W. Demtröder, Laser Spectroscopy, 2nd edn (New York: Springer) 1996. 